\title{
PROBLEMÁTICAS DE LA MUJER Y NUEVAS PERSPECTIVAS DESDE LA COMPRESIÓN DE LA MÍSTICA DE LA MASCULINIDAD EN RELACIÓN A LA VIOLENCIA DE GÉNERO.
}

INÉS PORTILLO

EMILIA MORENO

E.U. Trabajo Social. Universidad de Huelva.

\section{INTRODUCCIÓN}

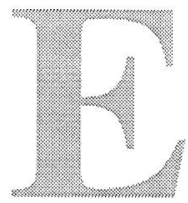

studios recientes en las diferentes áreas de conocimiento en la Universidad, y en otras instituciones, están generalmente orientados a conocer la realidad de numerosos aspectos de la situación de la mujer, como el análisis de los cambios de comportamiento en relación a los malos tratos y cuáles son las actuaciones que pueden favorecer la plena integración laboral en paridad con el hombre, junto al reconocimiento del valor económico del trabajo no remunerado, analizando los resultados con relación a la intervención social. ${ }^{1}$ Existe preocupación por la mujer en el tema de la violencia asociada al género y se hacen esfuerzos para avanzar, pero es muy lento el proceso hacia la igualdad real.

Es incuestionable el precio que pagan las mujeres, aunque también los varones sufren las consecuencias de ese proceso social. Contamos con pocas investigaciones que analicen cómo vivencia el hombre su proceso de desarrollo, la problemática que genera el sexismo en el hombre y cuáles son las exigencias que le impone la socialización de los roles sexuales, que se traducen en experiencias «con pérdidas»

\footnotetext{
Ver, entre otros, Vílchez Martín, L.F., 2000: «Cambios psicosociales en las mujeres que trabajan fuera de casa como variable determinante en la educación familiar», en tesis doctoral, «Educación familiar: los constructos implícitos en los que basan los padres la educación de sus hijos», UNED, Madrid (Tomo I, pp.260-277), Moreno, E., 2000: "La transmisión de modelos sexistas en la escuela», en «El harén pedagógico. Perspectiva de género en la organización escolar», Biblioteca de Aula, Barcelona (pp. 11-32), García, S., 2001:«La asignación de valor para el trabajo doméstico. Una lectura de la contabilidad satélite», en Jornadas «Tiempos, trabajo y género», U. de Barcclona (ponencia policopiada).
} 
como consecuencia de una socialización sesgada sexualmente, y que estimamos conllevan también violencia en su desarrollo pisococial. En relación a los hombres, hay necesidad de hablar de las «pérdidas» que el proceso de socialización de la masculinidad conlleva para él, sobre todo con relación a los aspectos femeninos de su ser, fuertemente aplastados, que tiene como una de sus consecuencias u objetivos, según se mire, el predominio de la esfera laboral en detrimento de la personal, de la vivencia de afectos y sentimientos y como cuidador del hogar y la familia.

La relación del ser humano y el trabajo ha estado siempre marcada históricamente por la división sexual. Sobre todo, como consecuencia de la revolución industrial y del crecimiento urbano, el trabajo se asume como una responsabilidad masculina. A la mujer, dotada biológicamente para la maternidad, se le asigna lo que se ha denominado el dominio de la «esfera privada»: el hogar, la familia, el cuidado de la prole... La «esfera pública» (la actividad pública, la política, el trabajo remunerado...) es el espacio masculino: el hombre es el encargado de salir de casa para ganarse la vida, para realizar el trabajo que proporciona remuneración. Se establece, de esta forma, una marcada diferencia entre trabajo productivo (remunerado) y trabajo reproductivo (el cuidado del hogar y la familia). Desde estas realidades podemos decir que, aunque la situación de la mujer y su papel a lo largo del tiempo ha ido cambiando, en paralelo a como lo ha hecho la familia y la sociedad, ha estado en una situación de desigualdad.

El acceso a la cultura, al voto y al trabajo, conquistas de la sociedad en los dos últimos siglos, fue posible para las mujeres con posterioridad a los hombres. La defensa de los derechos de las mujeres se hicieron públicos a la vez que la lucha por los derechos civiles de los grupos oprimidos, de las personas de raza negra, entre otros colectivos. El cambio más importante que les permitió situarse en el lugar adecuado, en paralelo con el hombre, fue debido, sobre todo, a los avances sanitarios logrados en el siglo XX y, concretamente, a la puesta en marcha de los métodos de planificación familiar, que liberaron a las mujeres, por un lado de la carga que conllevaban las altas tasas de natalidad y por otro del alto índice de mortalidad asociado a lo anterior. El acceso a la educación y al trabajo en igualdad de condiciones ha sido una conquista más legislativa y normativa que puesta en práctica en la realidad. La existencia de programas específicos para la mujer es un ejemplo de la necesidad de políticas discriminatorias positivas por las situaciones de violencia en diferentes grados que tienen las mujeres; los continuos malos tratos y la ausencia de la mujer en equidad con el hombre en puestos de responsabilidad - pese a los mejores resultados académicos que los varones- y en la política son una muestra de la necesidad de 
avanzar para hacer realidad la justicia social y para erradicar las desigualdades (Girela y Gallego, 1996:681 y ss.).

\section{CAMBIOS SOCIALES Y SU REPERCUSIÓN EN LA ASUN- CIÓN DE ROLES.}

Como consecuencia de los cambios ocurridos, hay un debate intenso y necesario sobre cuál va a ser el papel del varón en la sociedad actual donde su rol tradicional de trabajador por excelencia y alimentador de la familia se está desvirtuando, se desvirtúa de hecho. Por supuesto que hablamos en general, ya que no «todos los hombres son iguales» ni se tienen por qué sentir reflejados en lo que decimos. Estamos hablando de una tendencia. Como plantean Padilla y Moreno, en cita de Morgan, Skovholt y Orr (2000:308), el hombre «no tiene a dónde ir». Su evolución y sus posibles opciones, diferentes a las que tradicionalmente se le asignaban hace que, cuando quiere dedicarse al cuidado del hogar o aspirar a una profesión tradicionalmente considerada «femenina», es considerado un bicho raro, estando tan afectado como la mujer por los estereotipos sociales. Lo que se denomina la «mística masculina» es un peculiar sistema de creencias y valores que ejerce una presión directa en el desarrollo personal masculino. Sus implicaciones se pueden resumir en una superioridad de los hombres sobre las mujeres, en la manifestación de poder, control y dominio, esenciales en la masculinidad, y en evitar las emociones, los sentimientos, la vulnerabilidad y la intimidad, ya que son características femeninas. Desarrollar su carrera profesional y tener éxito económico son, finalmente, las medidas del logro del ser masculino. Si no acepta estos valores es castigado socialmente, y esto va asociado a una fuerte emoción negativa hacia los valores, actitudes y comportamientos femeninos. La mística masculina le conduce, por lo mismo, a afrontar el rol de trabajador desde una peculiar concepción. Tiene que triunfar profesionalmente porque si no es un fracasado. Tanto el joven que no ha triunfado como la mujer de éxito están expuestos/as a la marginación social. Han elegido un camino que la sociedad no está dispuesta a reconocer y valorar plenamente, ya que no coinciden con los papeles que socialmente se les tienen asignados. Levinson y otros, en cita de Padilla y Moreno, son los que más han trabajado y estudiado este fenómeno desde una perspectiva evolutiva. En este estudio de las fases del desarrollo personal y profesional de los hombres, se analizan los procesos de integración de varias polaridades a lo largo de la vida, como son la juventud y la vejez, la destrucción y la creación, y también la polaridad de la masculinidad y la feminidad. Cuando el hombre es joven no es capaz de integrar ambos polos por un conjunto de razones que justifican esta incapacidad 
para la integración: las tradiciones culturales, la inmadurez personal, los condicionamientos biológicos $\mathrm{y}$, sobre todo, la necesidad de dedicar las energías a otras tareas vitales más importantes (conseguir una ocupación y formar una familia). Hacia los cuarenta años surgirá una nueva estructura vital en la que reconocerá los aspectos femeninos de sí mismo. Es pues, según el estudio que seguimos, en la transición en mitad de la vida, entre los 40 y 45 años, cuando se consigue el equilibrio entre los polos masculino y femenino. Esta integración no es solamente cuantitativa: «reducir» uno y «aumentar» la otra. Se trata de construir nuevas relaciones en las relaciones arquetípicas que representan ambos polos. Esto supone modificar toda la estructura vital anterior, desde que comenzó a ser adulto joven, y trabajar en la construcción de una nueva en la que lo femenino no sólo tiene más participación, sino que no será inhibido. Cuando esto sucede, el hombre maduro es más capaz de combinar creativamente las dimensiones masculinas y femeninas de su yo para utilizarlas en su trabajo, en sus relaciones personales, en su intimidad, y, en general, en todas sus experiencias vitales. Algunos resultados del cambio son una mayor disposición a trabajar de forma colaborativa con la mujer, el establecimiento de relaciones de pareja más maduras y una mayor capacitación para convertirse en mentor, incluyendo ahora a las mujeres como posibles protegidas.

Desde este análisis, podemos concluir que en el proceso de desarrollo personal y profesional, en el proceso de desarrollo vital de toda persona, tanto el hombre como la mujer, cada uno tiene sus propias «contrapartidas» o pérdidas», que son manifestaciones de diferentes grados de violencia, siempre mayores en el caso de las mujeres. En el caso masculino hay una pérdida de la sensibilidad, los sentimientos y la solidaridad en pro de una mayor agresividad profesional, poder, control y dominio, dando respuesta a lo esencial de la «mística masculina». Es la imagen del hombre caracterizada por la agresividad, la toma de decisiones y la competitividad. Frente a esto se sitúa la imagen femenina asociada a la ternura, a la sensibilidad y a la responsabilidad. A la mujer se le asigna pues lo relativo a la maternidad y el cuidado, se le considera como emocional y con tendencia a tomar sus decisiones sobre la base de los sentimientos. Esto explicaría en cierta forma cómo la mujer está detrás de múltiples problemáticas sociales, desde nuestra experiencia profesional en el Trabajo Social en distintos campos: con familias que sufren paro crónico, en los casos de toxicomanías, en grupos con especiales dificultades, por su pertenencia a minorías sociales, cuidando a personas mayores, enfermos o discapacitados, y siempre asumiendo tareas y responsabilidades sin que sea posible compartirlas, y sin ser ella la causante, en la mayoría de las veces, de dichas situaciones. No hay frecuentemente mujeres toxicómanas, al menos en igual proporción que hombres; igual ocurre con las reclusas; son muy raros 
los casos de predelincuencia juvenil femenina; no hay apenas mujeres entre el colectivo fuertemente marginado de los Sin Techo, y, así podríamos seguir. La mayoría de las demandas que se reciben en el sistema de Servicios Sociales, en los Servicios de Salud o en cualquier otro servicio las plantean mujeres. Estarían las mujeres en todo lo relativo al cuidado, al mantenimiento de las personas en espacios relativos al hogar o cercanos a él.

La participación de la mujer en trabajos femeninos y masculinos sin que todavía los hombres hayan hecho lo correspondiente con los del otro sexo, nos ha llevado a una doble jornada o "jornada interminable", que sigue siendo signo de explotación y abuso, y que se disfraza argumentando que es una opción personal. Las cargas familiares son asumidas casi de forma exclusiva por las mujeres, limitándose los hombres a "ayudar", siendo oportuno a este respecto citar lo que dice Amando de Miguel: "la naturaleza ha hecho que las mujeres traigan a los hijos a este mundo, pero el que ellas sean también quienes tengan que alimentarlos, vestirlos y cuidarlos es ya un invento de la cultura humana y si se quiere una cómoda justificación de la organización androcéntrica de la vida". ${ }^{2}$

Esta vivencia de los roles en conflicto se comprueba en numerosos estudios, entre ellos, el citado al comienzo de esta comunicación de Vílchez (2000), donde el grupo de mujeres trabajadoras de clase media analizaban las dificultades para compatibilizar ambos roles -cuidado de la familia y trabajo-.

\section{PROBLEMÁTICAS MÁS GRAVES.}

Pese a que la Constitución española ha consagrado la igualdad de derechos -arts. 9.2 (corresponde a los poderes públicos promover las condiciones para que la libertad y la igualdad del individuo y los grupos en que se integra sean reales y efectivas; remover los obstáculos que impidan o dificulten su plenitud y facilitar la participación de todos los ciudadanos en la vida política, económica, cultural y social) y 14-de todos los ciudadanos, sin discriminación de sexos, y el derecho a la integridad y a la seguridad, aún persiste el problema social de los malos tratos, la violencia de género que se dice, pero que es especialmente de un sexo sobre el otro, del hombre sobre la mujer. En términos generales, se habla de $10 \%$ de violencia, como término medio, y todos los autores coinciden con los datos anteriores (VV.AA., 1991:35), y de entre un $5 \%$ y un $10 \%$ sólo de denuncias sobre los casos reales; en los

2 Para ampliar, ver datos del Instituto de la Mujer, «Diferencias en el uso del tiempo según sexo» http://www.mtas.es/mujer/organigr.htm. 
casos de mujeres muertas por los malos tratos conyugales, que parece que es fácil de controlar, se mantienen también diferencias según las fuentes sean del gobierno o de las asociaciones -en el año 1999, el gobierno daba la cifra de 33 y la Federación de Mujeres Progresistas y la Federación de Mujeres Separadas y Divorciadas 59 (Pérez-Díaz, Chuliá y Valiente, 2000:137)- . A estas cifras hay que sumar la tasa de suicidio femenino, puesto que entre el $20 \%$ y el $40 \%$ de las mujeres que lo llevaron a cabo habían estado sometidas a situaciones de maltrato.

Este problema social se produce en todas las clases sociales, independientemente de los niveles culturales o económicos y con todas las ideologías, siendo las más afectadas las amas de casa condicionadas por la dependencia económica, el desconocimiento de sus derechos y el aislamiento. Sus consecuencias, en general graves, van a depender de la estructura de la personalidad de cada mujer, de las características sociales, económicas, etc. La mayoría de ellas presentan trastornos psicológicos con un alto índice de angustia y/o depresión, con sentimientos de autodesvalorización y de inseguridad, necesitando apoyo psicológico que les ayude a tomar conciencia de su problema y adquirir la capacidad para afrontarlo. A estos daños hay que añadir los causados a los hijos que viven en estos ambientes de violencia y que tienen gran probabilidad de interiorizar comportamientos que perpetúen el modelo vivido.

Estas consecuencias son comunes también en casos de los maltratados. De nuestra experiencia en el Servicio Andaluz de Salud, no olvidamos el caso de un hombre maltratado por su mujer, al que le habían separado físicamente y aislado de las hijas, de su esposa, por supuesto, y de los amigos, reproduciendo la misma situación que en el caso de las mujeres maltratadas, de desvalorización, sentimiento de culpabilidad, en este caso con el añadido de tener una enfermedad grave, lo que aumentó más el desprecio y la separación de él. Sus ausencias intermitentes por causas laborales habían hecho que este hombre se agarrara, psicológicamente, a su mujer, como el único nexo de unión con el mundo, idealizándola, y cuando ya, tras tiempo pasado de sufrir desprecios y todo tipo de humillaciones, decidimos remitirlo al Centro de la Mujer, nos dijeron que no atendían a hombres. Hablamos de plantear una separación a la esposa y se echó a llorar y no quiso hacerlo. El Centro de la Mujer proporcionó el nombre de una abogada de familia y se derivó hacia allí el caso. A los tres meses, en coordinación con ella, se consiguió que aceptara que se mandara una carta a su mujer planteando la separación. Es por este motivo por el que, desde nuestra experiencia deberían ser Servicios Sociales para la Igualdad, ya que existen también problemáticas similares a las descritas en algunos hombres, pero 
su número es aún tan pequeño en relación al de las mujeres que no se ha dado ese paso, al menos en los Servicios Sociales Especializados.

Una investigación de Jiménez Casado, C., sobre «Malos Tratos conyugales a mujeres en el área de Sevilla», define la violencia como "algún acto que lleva a la víctima a hacer algo que ella no quiere hacer, le impide hacer algo que ella desea realizar o que le hace tener miedo", y desde un punto de vista más amplio «una persona es violentada cuando se hace daño a su psique, a su cuerpo, a su dignidad, o a su habilidad para gobernarse a sí misma...» (1996:20).

La violencia doméstica o abuso familiar no son sólo las graves lesiones que producen los ingresos en hospitales, ya que ésta es sólo una parte de una amplia variedad de formas de violencia que no llegan a conocerse públicamente porque se consideran violencia "normal" dentro de la vida familiar, por la existencia de pautas sociales patriarcales que todavía persisten entre nosotros. Las normas culturales que consideran la familia "como algo privado" donde lo que ocurra "tras las puertas cerradas" del hogar no debe ser contado a "extraños" estimulan indirectamente la violencia doméstica. Mujeres que han mantenido relaciones de violencia y abusos de pareja reconocen que, en general, no conocían ninguna forma de escapar de ella ni hacían confidencias de su situación de víctimas a otros, ya que eso significaría «revelar asuntos familiares» $(1996: 15)$.

Además de las teorías feministas que subrayan la importancia del hecho de la subordinación de la mujer como elemento básico en el problema que estamos tratando, hay tres causas fundamentales que lo pueden explicar:

1. Estaría en la propia estructura familiar que, al no poder afrontar adecuadamente las graves situaciones de estrés, desempleo, problemas de salud, inseguridad económica y otros, produce violencia entre sus miembros.

2. El alto grado de aceptación social de la violencia dentro de la familia, ya sea porque dentro de ella se admite como «bueno» cierto grado de violencia, el castigo o la presencia de la misma es vista con naturalidad, o en el exterior, donde la sociedad y los medios de comunicación «socializan» la violencia continuamente.

3. Gira en torno a teorías de origen psicológico. Unas porque entre los maltratadores se da con frecuencia comportamientos psicopatológicos y dificultades para afrontar el estrés. Otras porque se apoyan en la Teoría del Aprendizaje Social, donde el modelo de familias con conductas agresivas tiende a repetirse.

Finalmente, los huecos que no se llenan con estas explicaciones -no todos los que han vivido ambientes familiares maltratantes son a 
su vez maltratadores- buscan en la necesidad motivacional del hombre por el poder la causa de que se manifieste el abuso conyugal como espacio de asertividad. Los estudios que analizan la personalidad del agresor sugieren también que son personas que manifiestan sus emociones deliberadamente teniendo en cuenta el efecto social que las mismas provocarán. Explicaría esto en parte la doble personalidad que mantienen, ya que inicialmente muestran conductas sociales apropiadas, pero cuando se sienten amenazados emergen los rasgos negativos inherentes a su personalidad con demostraciones de violencia e intimidación. Suelen tener un sentido de la realidad distorsionado lo que les lleva a interpretaciones erróneas. Esta interpretación proporciona la base para conductas alternantes encontradas entre los sujetos maltratadores. En paralelo con lo anterior la mujer estaría «entrenada» para no responder de la misma manera ante una acción violenta (Jiménez Casado, 1996: 36 y ss.). Las teorías del conflicto también tratan de analizar las causas de la existencia y la persistencia de la violencia.

Lo que está demostrado es que no existe una personalidad previa que nos indique que una mujer tiene más posibilidades de sufrir maltrato que otra, según estudios realizados en Europa y en EEUU. También reflejan que las mujeres suelen ser de mayor nivel cultural que su marido y que el maltrato puede ser una forma de someter y rebajar a alguien superior. Hay entre ellas un porcentaje amplio de mujeres poco seguras de sí mismas y que realizaron un matrimonio joven.

Las consecuencias son iguales para todas, produciéndose un cambio importante en la personalidad, inhabilitándolas cada vez más para defenderse, para escapar, para funcionar eficazmente dentro o fuera del hogar. La reacción emocional se manifiesta con depresión, confusión, vergüenza, impotencia y un miedo paralizante, lo que los psiquiatras llaman «estado emocional plano». Esto hace que cuando van a hacer la denuncia sean lacónicas, inexactas u omitan detalles, y digan «me pegó» en referencia a los muy numerosos golpes con la correa, o «me empujó» cuando intentó matarla al tratar de arrojarla desde el balcón... Eso confunde a la policía y evitan hablar de maltratos sexuales y de violación. Viven por imposición del marido un aislamiento cada vez mayor.

\section{OTRAS PROBLEMÁTICAS SOCIALES Y COLECTIVOS DE RIESGO.}

La violación forma parte del maltrato y la violencia en el ámbito doméstico, y aumenta de forma considerable su gravedad y los trastornos psicológicos de la víctima al ser el agresor una persona cercana: 
duerme con él, en el caso de la pareja, o es su padre, hermano o tío, y, en estos casos y dentro del contexto de maltrato habitual, no suele ser denunciado. A nivel mundial, Ios datos de la última Conferencia de Pekín hablaban de que una de cada seis mujeres es violada en su vida.

Otra de las problemáticas en relación al género es el acoso sexual, que afecta a un $18 \%$ de mujeres, siendo lo preocupante el hecho de que un $35 \%$ de las afectadas toma la inevitable decisión de cambiar de trabajo. Un $28 \%$ se trasladan de puesto o de turno cuando tienen oportunidad. Sólo un 3\% lo denuncian legalmente, pierden el empleo y el pleito normalmente no prospera. Algunos hombres se reconocen en situaciones de acoso leve, pero apenas hay casos, y sólo lo perciben si está unido a una situación molesta, de discriminación laboral, personal, pero no se sienten objeto sexual. Al igual que ocurre con la violencia doméstica, no hay un perfil que defina a la mujer que sufre acoso, pero el $41^{\prime} 4 \%$ son separadas o divorciadas, y también es más frecuente entre quienes tienen mayor inestabilidad laboral, ya que el $27 \%$ de quienes lo producían pertenecían a este grupo (El País, 2001-02-02:31).

Hay un problema que afecta a las mujeres a nivel mundial, es la pobreza, fundamentalmente asociada a la mujer, teniendo por ello mayores riesgos de explotación, abuso y malos tratos (de los 1.300 millones de pobres absolutos, el $70 \%$ son mujeres). Las mujeres forman parte de las nuevas pobrezas, localizadas en determinados grupos de población que en nuestro país lo forman viudas, mujeres separadas, divorciadas, adolescentes con hijos no deseados, mujeres en la economía sumergida, mujeres inmigrantes, encarceladas y abuelas con nietos a su cargo y escasos recursos económicos. Son, sobre todo, mujeres jóvenes con cargas familiares -familias monomarentales- y sin pareja estable, y todas las que sufren alguna toxicomanía, también las mujeres de alcohólicos y las mujeres pertenecientes a otras culturas que son minorías étnicas en nuestro entorno (emigrantes, gitanos,...), junto a mujeres que están en la prostitución por diversas causas y procedencias.

La prostitución es un problema complejo y difícil de abordar que afecta a la mujer, también a menores y, en menor medida, a los hombres. Es el signo máximo de explotación y violencia humana. Según la experiencia de Cáritas (Cáritas, 2001:9), la pobreza es la que lleva a muchas mujeres y niñas a la prostitución. Es tan fuerte el movimiento económico que proporciona que hacen falta voluntades políticas muy claras y decididas para evitar la explotación sexual. El comercio internacional de personas engloba también el turismo sexual, tan de moda hoy en día. En Europa, el tráfico de mujeres para la explotación sexual constituye la mayor categoría de inmigrantes indocumentadas, “... el transporte de mujeres puede para fines de explotación 
sexual..., puede ser hecho con entrada legal o ilegal..., en calidad de "bailarina..., puede ser una tapadera para ejercer la 'prostitución") (Comisión Europea, 1998:7).

Dona Hughes, coordinadora de la ONG Coalición contra el Tráfico Sexual, ha denunciado que la industria mundial del sexo recauda, anualmente, más de 10 billones de pesetas. En España, se calcula que el 80 por ciento de las mujeres que ejercen la prostitución son extranjeras, la mayoría de ellas esclavizadas por redes de traficantes. El Primer Foro sobre la Prostitución, celebrado en Sevilla en octubre pasado, alertaba sobre el hecho de que el tráfico de mujeres con fines sexuales es más rentable que el tráfico de armas o de drogas. "Existe un gran marketing detrás de la venta de seres humanos; los Estados no son proxenetas pero son responsables al no aplicar todos los medios para evitarlo" dijo Wassyla Tamzali, directora del programa para la promoción de la condición de las mujeres del Mediterráneo, de la UNESCO (El País, 200010-02: 5).

Hay un colectivo especialmente maltratado y es el de las toxicómanas, muchas veces por inducción de la pareja, y que está en la prostitución por su adicción. Es doblemente maltratada y marginada por su situación. Es un grupo pequeño, pero son víctimas cada vez menos visibles y muy difíciles de recuperar. Los escasos estudios sobre esta población las muestran con tal cantidad de cargas que tienen que realizar un esfuerzo titánico para volver a la normalidad. Sus historias comienzan en su propia familia: tienen más antecedentes de abusos sexuales y maltrato que las que sufren violencia doméstica. Su adicción se ve como una mayor transgresión social y las deja sin apoyo familiar en el $90 \%$ de los casos. «La mujer siempre convive con el adicto; en cambio ellos vuelven a casa con su mamá». El desarraigo familiar está en la base del inicio al consumo por la pareja; hay una coadicción, una relación simbiótica de maltrato, con la pareja y con la droga. Los hijos son el motor para iniciar el tratamiento y las terapias les ayudan mucho a normalizar las relaciones familiares. El índice de paro entre ellas es muy alto y los trabajos que realizan son casi siempre de tipo marginal. Hasta en un $90 \%$ ejercen la prostitución por causa económica. Asegurarles las necesidades básicas es fundamental antes de iniciar cualquier tipo de intervención de ayuda y de apoyo (EI País, 21-2-1999:14).

El aborto es otra de las problemáticas de las que hay que hablar en relación a la mujer y la violencia que padece. La libertad de reproducción es hoy en nuestro país una quimera, algo que está muy lejos de poder ser alcanzado. La brusca caída de la natalidad en nuestra sociedad no se debe ni a políticas sanitarias adecuadas, que contemplen mayor apoyo a la planificación familiar, ni a la existencia de políticas 
natalistas. La doctora Andrés (2000) explicó, en las « $1^{\text {as. Jornadas In- }}$ ternacionales sobre el aborto en la salud reproductiva», que España con una tasa de fecundidad de las más bajas, l'16 por mujer, lo único que hace es apoyar y subvencionar a grupos que promueven y apoyan a «la familia». ¿Dónde queda el derecho a la opción por la maternidad?

Han disminuido los servicios gratuitos de planificación familiar. Las restricciones en este campo conllevan un aumento en el número de abortos y no de partos, fenómeno ya comprobado cuando hay políticas restrictivas de métodos anticonceptivos. Según la Consejería de Salud, las Interrupciones Voluntarias del Embarazo-IVEs-declaradas y realizadas en los centros acreditados y hospitales en el año 1998 en nuestra comunidad fueron de 8.581 , datos que se refieren a mujeres andaluzas, residentes en nuestra comunidad. De esta cifra hay que destacar que 38 de ellas lo fueron a menores de 14 años y que el $49^{\prime} 94 \%$ fueron IVEs realizadas a mujeres entre los 20 y 29 años (Junta de Andalucía, 2000:223). Una encuesta del Instituto Nacional de Estadística refleja que en la misma banda de población y sexo con 2'35 millones de mujeres, 270.000 han recurrido a la IVE y 20.000 de ellas dos o más veces.

Es importante destacar que Holanda, país con 30 años de libertad de elección en relación a la maternidad y una red de clínicas donde atendieron también a muchas españolas, ostenta la tasa real de abortos más baja del mundo, gracias a un ejemplar sistema sanitario, de educación sexual y de planificación familiar (Stlzenburg, 2000).

\section{LA PROTECCIÓN JURÍDICA DE LA MUJER Y LOS SERVI- CIOS SOCIALES ESPECIALIZADOS.}

Desde los Servicios Sociales se trabaja para disminuir las condiciones de desigualdad, considerando a la mujer como sujeto de prestaciones sociales, ya que esas condiciones la dejan en situación de grave precariedad en relación al fenómeno grave de la violencia doméstica. Los últimos datos en nuestro país hablaban de que cada media hora una mujer es maltratada en su medio familiar, lo que supone al mes tantos actos de maltrato como días tiene el año, es decir 360.

Desde la Unión Europea se han realizado tres programas de acción para la igualdad de oportunidades y el cuarto ha finalizado en el 2000 , para reforzar la igualdad en combinación con una estrategia de crecimiento económico orientada hacia la intensificación del empleo, al desarrollo de iniciativas para aumentar la flexibilidad, el trabajo a tiempo parcial, mejorar ofertas de cualificación y favorecer la creación y 
adquisición de empresas por mujeres. De 2001 a 2006 se está desarroIlando la iniciativa EQUAL (BOE de 17 de abril de 2001), instrumento ejecutivo de la Estrategia Europea para el Empleo, directriz que surgió del Consejo Europeo de Luxemburgo. Es una iniciativa que tiene cuatro grandes objetivos relativos al empleo, uno de los cuales es el fomento de la igualdad de oportunidades de hombres y mujeres. La asignación presupuestaria para España es de 85.490 millones de pesetas (algo más de 35.300 millones de pesetas en la primera etapa del 2001-2003), y todos los proyectos que se financien con cargo a ella, tienen que incorporar objetivos y actuaciones concretas en materia de igualdad de oportunidades entre hombres y mujeres en cada proyecto (Orngsocial, núm. 3, 2001:24-26).

A nivel nacional el Instituto de la Mujer ha elaborado los Planes para la Igualdad de Oportunidades, habiendo terminado el año pasado el III Plan para la Igualdad de Oportunidades entre Mujeres y Hombres (1997/2000), realizándose la evaluación en este momento. En líneas generales pretendía:

- introducir la igualdad de sexo en todas las políticas activas,

- impulsar el avance de la mujer en todas las esferas de la vida social,

- apoyar especialmente los ámbitos de economía productiva y de toma de decisiones.

Se da una gran importancia al empleo, pues la mayoría de las medidas van dirigidas a esta área y también responden, en su conjunto, a los compromisos adquiridos en la IV Conferencia Mundial de Naciones Unidas celebrada en Pekín. En esta conferencia se asumen objetivos en las áreas de educación, salud, economía y empleo, en la toma de decisiones, en los medios de comunicación, el medio ambiente, la violencia, la exclusión social, las mujeres rurales y la cooperación internacional, incluyendo, cada una de ellas, medidas concretas a desarrollar.

En este Plan se incluye el concepto de «maimstreaming» ${ }^{3}$ procedente del último plan europeo, que supone promover la igualdad de oportunidades entre hombres y mujeres en todas las actividades y políticas, y en todos los niveles, es decir, atravesar horizontal, vertical y transversalmente todos los ámbitos de la sociedad con este propósito.

\footnotetext{
GABINETE DRH\&T, 1999: Mainstreaming (de género). El concepto mainstreaming fue utilizado por primera vez en el transcurso del Tercer Programa Comunitario de acción a medio plazo, pero no es hasta 1995, durante la celebración en Pekín de la IV Conferencia Mundial sobre las Mujeres, que este concepto alcanza la dimensión de una gran estrategia para la igualdad de oportunidades entre las nujeres y los hombres, a escala global. Pero, ¿qué significa el término anglosajón mainstreaming?. Main es una adjetivo que en inglés significa principal. Stream es un sustantivo que quiere decir corriente o torrente (de un río, por ejemplo). Ing es una desinencia verbal que se utiliza para formar el gerundio. Así pues, el término mainstreaming (que no tiene traducción literal), a modo de metáfora, quiere significar «convirtiendo en corriente principal».
} 
Especialmente importante es el primer Plan de Acción sobre la Violencia contra la Mujer, que fue aprobado el 30 de abril de $1998^{4}$ y que finalizó el año pasado, y en función de los resultados obtenidos se ha elaborado el $2^{\circ}$ Plan Contra la Violencia Doméstica, que estará en vigor hasta el año 2004. Según Dancausa, Secretaria General de Asuntos Sociales, será también interministerial -Trabajo y Asuntos Sociales, Justicia, Sanidad, Educación e Interior-, contempla, junto a medidas preventivas y educativas, como prioridades, lograr mayor protección para las mujeres agredidas. Se refuerza la red de centros de acogida, se impulsa la mejora de la formación de quienes atienden estos casos y se mejorarán los dispositivos policiales que atienden estos casos. Según cita la policía, se estima en un $60 \%$ el número de delitos cometido en el ámbito doméstico no denunciados, incluyendo los relativos a menores y el acoso sexual (2001:33).

Sus resultados no fueron los esperados, ya que el primer año del plan aumentaron las muertes en un $25,7 \%$, aunque en el lado positivo se sitúa el aumento de denuncias en un 14\% (EL PAIS, 15-2-2001, pp. 33).

Este II Plan contra la Violencia no ha admitido las propuestas de las asociaciones de mujeres juristas y de las asociaciones de mujeres, que habían realizado un estudio denunciando que el problema de la sanción penal de la violencia familiar es, no de ausencia de normas, sino de voluntad real de aplicación de las normas de los operadores jurídicos. Sí ha incluido las medidas legislativas sugeridas por otro informe del Consejo General del Poder Judicial, en relación a modificar la cantidad de absoluciones, rebajas de condenas o anulaciones de sentencias, reconocidas por él. No se ha elaborado una Ley Integral contra la violencia de género, que sí parece apoyar el PSOE, y se potencian las medidas de apoyo para la integración laboral de las mujeres maltratadas y se contemplan medidas preventivas y los servicios de mediación familiar (Orgnsocial, 20021:31).

Algunas de las conclusiones del Manifiesto de la Asociación de Mujeres Juristas (1998) basadas en el análisis de los procedimientos judiciales por causas de denuncias de maltrato son las siguientes, conclusiones que ponen de manifiesto cómo la violencia de género se practica desde el ámbito jurídico:

- En un 90\% de los casos el agresor era varón.

- Las denuncias son incompletas por que no se detallan, no hay una

4 Aprobado en Consejo de Ministros y según acuerdos adoptados en la Conferencia Sectorial sobre violencia de 20 de ese mismo mes, fue un plan integral por cuanto implicó a varios ministerios, con un presupuesto de 8.941.418.727 (Fuente: Ministerio de Asuntos Sociales). 
inspección ocular en el sitio de la agresión, ni fotos, ni testigos directos o indirectos...

- Los jueces tienden a calificar como faltas agresiones físicas y verbales entre parientes, aún cuando los hechos, por su gravedad, sean constitutivos de delito. Un $3^{\circ} \%$ de las denuncias tramitadas como faltas son amenazas de muerte que debieran haberse incoado por procedimiento abreviado. Un $3 \%$ de las denuncias de faltas, por su gravedad, deberían haber sido delito, lo que hubiera aumentado en un $70 \%$ los procedimientos abreviados, por violencia familiar. Los malos tratos habituales, denunciados por el $50 \%$ de las víctimas, carecen de aplicación práctica. Por ello, de 300 juicios de faltas sólo se siguen 3 ante el juzgado de lo penal y 1 ante la Audiencia Nacional.

La Junta de Andalucía ha desarrollado dos planes integrales para la Mujer. El II Plan Andaluz para la Igualdad de las Mujeres (19951997) está en proceso de evaluación. Contemplaba veintiún objetivos en relación a cinco áreas, relativas a:

1. Profundizar en el desarrollo de la legislación, información sobre sus derechos, apoyar la participación, la igualdad real en lo público y lo privado y eliminar la educación sexista y discriminatoria.

2. Favorecer el desarrollo de medidas concretas que favorezcan la igualdad, y mejoren la investigación en este campo.

3. Actuar para elevar el nivel de bienestar social, en relación a la salud en todas las etapas de la vida de la mujer, así como las medidas para apoyo a mujeres con cargas familiares no compartidas y también para las que sufren maltrato y violencia de género.

4. Desarrollo de todas las actividades tendentes a mejorar la situación sociolaboral de las mujeres, tanto en lo que se refiere a la formación para el empleo como el apoyo al autoempleo y a las mujeres empresarias.

La Consejería de Educación y Ciencia también ofrece, a través de los Centros de Educación de Adultos, formación a mujeres, e igualmente favorece y apoya los Seminarios e Institutos Universitarios de Estudios de la Mujer de las distintas Universidades andaluzas, que realizan un importante trabajo en el campo de la investigación en temas y problemáticas de mujer, organizando cursos, encuentros y jornadas, y favoreciendo la información, documentación y bibliografía específica en temas de género e igualdad.

A la vez que este plan se desarrollaba, el 17 de febrero de 1998 se aprobó el Plan de Actuación del Gobierno Andaluz para avanzar en la erradicación de la violencia contra las mujeres, que recoge las medidas aprobadas en el Parlamento de Andalucía y propuestas por las asociaciones de mujeres, en consonancia con el correspondien- 
te plan a nivel nacional. Entre la actuaciones realizadas, y en base a la medida número 7 del citado plan, está la puesta en marcha de un Procedimiento de Coordinación que supone, mediante diferentes pautas de actuación, una acción coordinada y global, con recomendaciones, instrucciones y directrices, que compromete a diferentes Consejerías -Presidencia, Gobernación y Justicia, Salud y Asuntos Sociales-junto al Tribunal Superior de Justicia y la Federación Andaluza de Municipios y Provincias, para conseguir una mejora y más eficaz acción pública contra la violencia de género (Junta de Andalucía, 2000:4,5).

\section{PERSPECTIVAS ANTE LA VIOLENCIA DE GÉNERO EN LA INTERVENCIÓN SOCIAL.}

En base a las Políticas de Servicios Sociales recogidos en el Informe Económico y Financiero del Presupuesto de la Comunidad Autónoma para el año 2001 tenemos en el capítulo de mujer como objetivos con relación a la intervención social:

- Incrementar la calidad de vida y la atención social a las mujeres.

- Fomentar la igualdad de oportunidades en el empleo.

- Superar las condiciones que limitan la participación política y social de las mujeres.

Si bien estos objetivos son muy genéricos, se concretan en continuar mejorando el ámbito legislativo, aumentar los recursos específicos, la red de CMI y todo lo que ayude a elevar el nivel cultural y la participación social, profundizar en el conocimiento de la falta de igualdad, la sensibilización ante él, y su reconocimiento, promover la sensibilización de hombres y mujeres para que compartan más igualitariamente espacios públicos y privados, fomentar una imagen pública de la mujer en consonancia con su realidad actual (ver columna del País....), continuar con estudios e investigaciones sobre la realidad y problemáticas concretas, así como promover los estudios y opciones no sexistas, e incluir la educación afectiva y sexual desde los centros de enseñanza y analizar comportamientos individuales y sensibilizar sobre situaciones que pueden conducir al maltrato en mujeres y niños en el ambiente familiar.

En la intervención social hay que hacerse un replanteamiento, basándose en investigaciones que profundicen en el sistema de valores masculino y femenino y su incidencia en los comportamientos, preocupándonos de qué forma se elaboran los proyectos personales y vitales. Las expectativas no se cubren sólo con el desarrollo profesional, sino que debe incluir la formación para el tiempo libre, el ocio y para las responsabilidades de la vida cotidiana. 
Las mujeres manifestamos, por todo lo dicho, conflictos entre los roles de trabajadoras y cuidadoras. Hemos avanzado mucho en el plano cognitivo, en lo que sabemos que tenemos que hacer y debemos hacer, no tanto en el afectivo-dejar compartir la gerencia de los afectos y el cuidado de los hijos, pero lo que está a muy larga distancia en el cambio son las realizaciones sociales, y esto nos produce desajustes y continuos reajustes. Entre la frustración y la agresividad se sitúan muchas de nuestras vivencias, dirigiéndolas a veces contra la sociedad y el hombre, y otras contra nosotras mismas, con ansiedad y sentimientos de culpabilidad, o pensando que la situación mejorará. El lenguaje y su análisis encierra toda una variedad de pensamientos, repitiéndose continuamente independencia, igualdad, culpabilidad, insatisfacción, ansiedad, falta de tiempo, prioridades, trabajo, afectos, hijos, autoestima,... (Vílchez, 2000).

Hay razones para la esperanza, ya que desde el año 1991, en que empezaron los programas para la mujer, se ha producido un aumento en general del número de denuncias; la edad de las denunciantes ha bajado, así como también han disminuido el número de años que llevaban soportando los malos tratos al hacer la denuncia; es también importante el número de mujeres mayores que piden ayuda y hacen denuncias planteando la separación; las fuerzas y cuerpos de seguridad están actuando con mayor eficacia y sensibilidad y hoy es raro el caso, muy común antes, de convencer a la mujer para que retire la denuncia; y desde los servicios de salud mental también ha aumentado la sensibilidad por esta problemática, dándose cuenta de que detrás de múltiples patologías estaba como causa, un maltrato continuado, y si no se abordaba no se podía intentar curar la enfermedad. Hay que entender, terminaban recordando, que la aparente insensibilidad de quien soporta malos tratos no es tal sino consecuencia de una grave situación personal y que el camino a recorrer es lento, pero puede hacerse con apoyo y medidas concretas.

En síntesis, tratar de inducir cambios en los niveles de violencia conlleva cambios radicales en diversos aspectos relacionados con la cultura, y ahí entra la educación, los modelos en la familia, el grupo de iguales, la publicidad, los mensajes indirectos de la prensa, el cine y el contenido dedicado a actividades que se califican como de uno u otro sexo.

Estamos aislando en las políticas sociales contra la violencia a los hombres y las investigaciones demuestran, incluso, la existencia de un "machismo suave" que hace que se motive a la mujer a continuar con sus roles tradicionales y que se minimice y desprestigie cualquier actividad o profesión en la que las mujeres están situándose en igualdad con los hombres. Estas situaciones son consecuencia, entre otras cau- 
sas, de "ese abandono" institucional y social de los hombres, y de la falta de programas educativos y sociales que visibilicen las pérdidas del proceso de socialización del rol masculino y valoren los roles tradicionales de las mujeres.

Una sociedad más justa debe ser una sociedad más desarrollada económicamente, con una mayor redistribución de la riqueza y con una democracia más avanzada, más participativa y una sociedad más igualitaria. Feminismo, desarrollo humano, solidaridad y paz van unidos. Uno sin otro no pueden existir.

\section{BIBLIOGRAFÍA.}

A.M.U.V.I.: Asociación Asistencia a Víctimas de Agresiones Sexuales, 1999: Abusos sexuales a Menores, Dirección General de Atención al Niño, Junta Andalucía, Sevilla.

ALCOBENDAS TIRADO, M.P., 1983: Datos sobre el trabajo de la mujer en España, CIS- Centro de Investigaciones Sociológicas, Madrid.

ALEMÁN BRACHO, C., 1991: EI sistema público de servicios sociales en España, Impredisur, Granada, pp. 309-316.

ALCOBENDAS TIRADO, M. P., 1983: Datos sobre el trabajo de la mujer en España, CIS, Centro de Investigaciones Sociológicas, Madrid.

ALEMÁN BRACHO, C., 1991: EI sistema público de servicios sociales en España, Impredisur, Granada, pp. 309-316.

ALEMÁN BRACHO, C., y GARCÉS FERRER, J. (dirs.), 1996: Administración social: servicios de bienestar social, Siglo XXI, Madrid.

ALEMÁN BRACHO,C. y GARCÉS FERRER, J. (coord.), 1998: Política Social. Editorial McGraw Hill. Madrid.

ALEMÁN BRACHO,C., y PÉREZ SÁNCHEZ, M., 2000: Los servicios sociales en Andalucía, en Los Servicios Sociales en las Comunidades y Ciudades Autónomas, de Gutiérrez, A., y Gárces, J. (Coords.), Tirant lo Blanch, Valencia, pp.21-54.

ANDRÉS, A., 2000: Situación actual del aborto, 1as. Jornadas Internacionales sobre el aborto en la salud reproductiva, mayo, Sevilla, (documentación policopiada).

AYUNTAMIENTO DE HUELVA, 2001: Manifiesto Asociación de Mujeres Juristas, Taller sobre Mujer y Violencia, 16 febrero, documento de trabajo, Huelva.

BARAHONA GOMARIZ, M.J., 1994: Reflexión teórica de la puesta en marcha del programa de atención a la mujer prostituida, Revista Médicos del Mundo, Madrid (texto policopiado).

BUBBY, G., 1994: Historia de las mujeres en Occidente. Tomo V. Editorial Círcu- 
lo de Lectores. Madrid.

CAMARERO SANTAMARÍA, J., 1999: Los derechos sociales en España tras veinte años de Constitución, Revista Documentación Social núm. 114, Cáritas Española, Madrid, pp. 55-75.

CÁRITAS DIOCESANA, 1997: El acceso al Servicio Andaluz de Salud del colectivo de mujeres prostitutas de Huelva capital, Centro Ammar, $2^{\circ}$ estudio (documento policopiado), Huelva.

CÁRITAS ZARAGOZA, 1996: La prostitución en la calle, Cáritas Diocesana, Zaragoza.

CARITAS, 2001: Cáritas Española, Enero, Madrid.

CARRASCO, C. y RODRÍGUEZ, A., 1999: Trabajos y cuidados: Hacia la reorganización social del tiempo y el trabajo, Revista Servicios Sociales y Política Social, Consejo General de Colegios de Diplomados en Trabajo Social y Asistentes Sociales, núm. 45, ler.tte. Madrid, pp. 61-84.

CASTRO, M., 1984: Papel de las Asociaciones de Consumidores en materia de regulación económica y comercial, Revista de Estudios Sociales y de Sociología Aplicada. Madrid.

COBO, R., 2000: Multiculturalismo, democracia paritaria y participación política, VII Encuentro Andaluz de Formación Feminista, Baeza (Jaén) (documento policopiado).

COMISIÓN EUROPEA, 1998: Igualdad de Oportunidades entre mujeres y hombres en la Unión Europea, Informe Anual de la Comisión, Comisión de las Comunidades Europeas, «Igualdad de Oportunidades entre las Mujeres y los Hombres. Tercer Programa de Acción Comunitaria a medio plazo» 1991-1995.

CONFERENCIA MUNDIAL SOBRELAS MUJERES, 1995: Las españolas en el umbral del siglo XXI, Informe presentado por España, Beijing.

CREMADES, M.N., 1990: Programas de Servicios Sociales, Carpeta Mujer, Acción voluntaria, Ayuntamiento de Madrid.

CUADRADO TENO, J.,1990: Sistema Judicial. Trabajo Social en el ámbito de la justicia: análisis de la situación, Revista Servicios Sociales y Política Social, Consejo General, Madrid, pp. 58-67.

DELGADO MORALES, J.F. y MARTÍNEZ MAROTO, A. (comp.), 1998: Hacia los Servicios Sociales del año 2000. Ministerio de Trabajo y Asuntos Sociales. Madrid.

FERRÁN CASAS, 1991: Los servicios de bienestar social: justicia, en Trabajo Social. Conceptos y herramientas básicas, ICEBS, Barcelona, pp. 309-319.

FUNDACIÓN ESPRIU, 2000: Las mujeres, en pie de paz, Dosier, núm. 40, dic. 2000, Revista Compartir, Gramagraf, S.C.C.L., Barcelona, pp.19-38.

GABINETE DRH\&T, 1999: Política social IV. Políticas de igualdad entre hombres y mujeres, en Curso de Experto en Recursos Sociales Europeos y Nuevas Tecnologías. Aplicación práctica al Trabajo Social, Colegio Oficial 
de Trabajadores Sociales de Málaga (documento policopiado).

GAIL PHETERSON, 2000: El prisma de la prostitución, Talasa, Madrid.

GARCÉS FERRER, J., 1992: Los Servicios Sociales especializados: Mujeres en Administración social pública. Bases para el estudio de los servicios sociales, Alemán, C. y Garcés, J., Tirant lo Blanch, Valencia.

GIRELA, B.A. y GALLEGO, A., 1996: Los Servicios sociales en función del sexo. Servicios Sociales para la mujer, en Administración Social. Servicios de bienestar social, Siglo XXI, Madrid, pp. 681-703.

GUALDA, Ma . P., DELGADO, J.F. y RODRÍGUEZ, A. (coord.), 1995: Avances en Política Social. Diputación Provincial de Granada. Granada.

INSTITUTO ANDALUZ DE LA MUJER 1999: Violencia contra las mujeres, Manual de Formación, Instituto Andaluz de la Mujer, Sevilla.

INSTITUTO ANDALUZ DE LA MUJER, 1999: Violencia contra las mujeres. Ámbito judicial, Manual de Formación, Instituto Andaluz de la Mujer, Sevilla.

INSTITUTO ANDALUZ DE LA MUJER, 1999: Violencia contra las mujeres. Ámbito social, Manual de Formación, Instituto Andaluz de la Mujer, SeviIla.

INSTITUTO ANDALUZ DE LA MUJER, 1999: Violencia contra las Mujeres. Ámbito sanitario, Manual de formación, Instituto Andaluz de la Mujer, Sevilla.

INSTITUTO ANDALUZ DE LA MUJER y CONSEJERÍA DE TRABAJO E INDUSTRIA, 1997: Proyecto LIBRA de la iniciativa NOW: Módulo de Igualdad, del material didáctico. Formación de Formadores/as. Formación en Igualdad, Sevilla.

INSTITUTO ANDALUZ DE LA MUJER, 1996: Revista MERIDIANA, núm. 0, enero, Sevilla.

INSTITUTO ANDALUZ DE LA MUJER, 1999: El aborto, Revista MERIDIANA, núm. 12, (tema central) Sevilla, pp. 14-27.

IZQUIERDO ROJO, M.,1995: Mujer y sociedad en Avances en Política Social, Diputación Provincial de Granada, Granada.

JIMÉNEZ CASADO, C., 1996: Malos tratos conyugales a mujeres en el área de Sevilla, Instituto Andaluz de la Mujer, Sevilla.

JUNTA DE ANDALUCÍA, 2000: Anuario Estadístico de Andalucía 2000, Consejería Gobernación, Sevilla.

JUNTA DE ANDALUCÍA, 2000: Procedimiento de Coordinación para la Atención a las Mujeres Victimas de Malos Tratos y Agresiones Sexuales, Instituto Andaluz de la Mujer, $1^{a}$ reimpresión, julio, Sevilla.

LÓPEZ, M.L. 1990: La vida asociativa, fomento de las políticas sociales. En busca de un espacio social europeo, en Política social y participación, Documentación Social, Cáritas Española, núm. 80, Madrid.

MEDINA, C. de, 1985: Participación y Trabajo Social, Humanitas, Buenos 
Aires.

MINISTERIO DE ASUNTOS SOCIALES, 1991: Conducir el cambio estructural. El papel de las mujeres, Instituto de la Mujer, Madrid.

MORENO SÁNCHEZ, E., 2000: La transmisión de modelos sexistas en la escuela, en Santos Guerra, M. A., El harén pedagógico. Perspectivas de género en la organización escolar, Grao, Barcelona.

ORNG, 2001: Fundación Luis Vives, núm. 3, 2001:24-26.

PADILLA, T, y MORENO, E., 2000: El desarrollo profesional masculino: una perspectiva de género en la orientación profesional, en Revista de Educación XXI, Universidad de Huelva, pp. 301-312.

PARLAMENTO EUROPEO, 1996: Informe sobre la participación de los ciudadanos y de los interlocutores sociales en el sistema institucional de la Unión Europea. Documentos de Sesión del Bruselas.

PARLAMENTO EUROPEO, 1998: La Europa de los Ciudadanos. Oficina de Publicaciones Oficiales de las Comunidades Europeas. Luxemburgo.

PAYNE, M. 1995: Teorías contemporáneas del Trabajo Social, Paidós, Barcelona, pp. 275-282.

PÉREZ-DÍAZ, V., CHULIÁ, E. Y VALIENTE, C., 2000: La familia española en el año 2000, Visor dis. S.A., Madrid, pp.130-137.

PORTILLO MAYORGA, I., 2001: Mujer e investigación bajo el prisma del trabajo social, en La tarea de observar desde la perspectiva de género, Universidad de Huelva (en prensa).

PORTILLO MAYORGA, I., 2001: La mujer en la sociedad actual, en Guía práctica de los Estudios de las Mujeres, Instituto Andaluz de la Mujer (en prensa).

RAMOS, F.,1999: Trabajo Social con víctimas; tipos de intervención y aspectos metodológicos, Revista TS Difusión, núm. 17, Málaga.

REYZABAL, Ma V., y SANZ, A.I., 1999: Violencia contra las mujeres: prevención educativa, Suplemento, Revista, núm. 248, marzo, Cáritas Española, Madrid.

SECRETARÍA DE POLÍTICA SOCIAL, 1999: Formación en Igualdad de Oportunidades, Departamento de la Mujer de UGT-Andalucía, Marzo, Sevilla.

STLZENBURG RAMOS, A., 2000: Aplicación de la ley del aborto en juzgados y tribunales. Reflexiones desde la perspectiva médico-sanitaria, las. Jornadas Internacionales sobre el aborto en la salud reproductiva, mayo, Sevilla, (documentación policopiada).

TRIGUEROS GUARDIOLA, I, 1996: Manual de prácticas de trabajo social con las mujeres, Siglo XXI, Madrid.

URBEZ, L. 1989: Prostitución femenina en España, Revista de Fomento Social, núm. 176, Madrid.

VÍLCHEZ MARTÍN, L.F., 2000: Cambios psicosociales en las mujeres que trabajan fuera de casa como variable determinante en la educación fami- 
liar, en Educación familiar: los constructos implicitos en los que basan los padres la educación de sus hijos, Facultad de Educación, Universidad Nacional de Educación a Distancia, Tesis Doctoral, Tomo I, pp. 260-277, y Tomo II, pp. 52-65 (material inédito).

VV..AA, 1997: Exclusión social y trabajo social en Europa. Facilitando la inclusión, FITS, Federación Internacional de Trabajadores Sociales- Europa, (documento interno Consejo General de Colegios de TT.SS. y AA.SS.), Madrid.

VV.AA., 2000: La circulación de mujeres. Ciudadanía, migración y procesos de integración europea, V Jornadas sobre Formación Feminista, Baeza (Jaén), Instituto Andaluz de la Mujer (documento policopiado).

VV..AA, 1999: Estudio sobre la Mujer en el Sistema Socio-Sanitario, Revista Servicios Sociales y Política Social, Consejo General de Colegios de Diplomados en Trabajo Social, núm. 45, 1er.tte. Madrid.

VV..AA., 1991: Violencia contra la mujer, Ministerio de Interior, Madrid.

VV..AA. 1994: Plan Andaluz de Servicios Sociales, Consejería Asuntos Sociales, Sevilla.

http://www.mtas.es/mujer/organigr.htm

http://www.junta-andalucia.es/iam/direcc.htm

http://www.mtas.es/mujer/visualiza.htm 